\title{
The value of a statistical life under changes in ambiguity
}

\section{Han Bleichrodt ${ }^{1,2} \cdot$ Christophe Courbage $^{3,4} \cdot$ Béatrice Rey $^{5}$}

Published online: 24 April 2019

(C) The Author(s) 2019

\begin{abstract}
The value of a statistical life (VSL) is a key parameter in the analysis of government policy. Most policy decisions are made under ambiguity. This paper studies the effect of changes in ambiguity perception on the VSL. We propose a definition of increases in ambiguity perception based on Ekern's (1980) definition of increases in risk. Ambiguity aversion alone is not sufficient to lead to an increase in the VSL when the decision maker perceives more ambiguity. Our results highlight the importance of higher order ambiguity attitudes, particularly ambiguity prudence.
\end{abstract}

Keywords Value of a statistical life $\cdot$ Ambiguity Prudence $\cdot$ Smooth ambiguity model · Neo-additive preferences

JEL Classifications D81 $\cdot \mathrm{I} 18 \cdot \mathrm{Q} 51$

\section{Introduction}

Reductions in mortality risk are the main benefit of government regulation in the US and other developed countries (Viscusi 2018). This makes the value of a statistical life

Electronic supplementary material The online version of this article (https://doi.org/10.1007/s11166-01909296-3) contains supplementary material, which is available to authorized users.

Han Bleichrodt

bleichrodt@ese.eur.nl

1 Erasmus School of Economics, PO Box 1738, 3000 DR Rotterdam, The Netherlands

2 Research School of Economics, Australian National University, Canberra, Australia

3 Haute Ecole de Gestion de Genève, Carouge, Switzerland

4 University of Applied Sciences Western Switzerland (HES-SO), Delémont, Switzerland

5 Univ Lyon, Université Lumière Lyon 2, GATE UMR 5824, F-69130 Ecully, France 
(VSL), which is the most common metric to assess the effectiveness of such regulation, a key parameter in the analysis of public policy. ${ }^{1}$

A large literature has explored how the VSL changes with, amongst others, wealth (Hammitt 2000), the baseline mortality risk (Pratt and Zeckhauser 1996), background financial risk (Eeckhoudt and Hammitt 2001), age (Viscusi and Aldy 2003), health status (Hammitt 2002), and altruism (Andersson and Lindberg 2009). All these studies take the mortality risk (and changes therein) as known. However, in most real-life decisions mortality risks are not precisely known and are subject to ambiguity. For example, experts have conflicting opinions about the exact risks to human life posed by climate change. Most experts agree that these risks exist, but they disagree about their exact size, leading to ambiguous estimates.

Ellsberg's famous paradox (1961) showed that ambiguity affects people's behavior. Ambiguity aversion can account for several empirical puzzles that traditional economics has difficulty explaining. Examples include the stock market participation puzzle (the finding that many people do not buy stocks even though they should according to standard portfolio theory) ${ }^{2}$, home bias (the finding that people invest too much in stocks from their own country) $)^{3}$, and the low take-up of freely available genetic tests (Hoy et al. 2014). Ellsberg's paradox has spurred the development of a variety of new ambiguity theories (Ryan 2009; Gilboa and Marinacci 2016; Machina and Siniscalchi 2014). Of these new theories, the smooth model of Klibanoff et al. (2005) is most widely used in economic applications.

In this paper we explore the impact of ambiguity on the VSL under KMM's smooth model. Treich (2010) showed that when one decision situation is ambiguous but the other is not, ambiguity leads to an increase in the VSL of an ambiguity averse (smooth) decision maker compared with the situation in which there is no ambiguity. However, in real-world decisions both alternatives are usually ambiguous, although they may differ in their degree of ambiguity. For example, more research into climate change may lead to more precise estimates of the risks to human life even when the exact risks remain unknown. Our main results (Results 2 and 3) show that in this more general case it is no longer true that an ambiguity averse decision maker will always have a higher VSL when he perceives more ambiguity. The effect of general changes in perceived ambiguity is more complex than the comparison between ambiguity and no ambiguity may suggest and requires information about higher order ambiguity preferences. In particular, our results show the importance of ambiguity prudence.

The paper is structured as follows. In Section 2 we give background and derive the VSL under risk. Section 3 then introduces ambiguity and derives the VSL under KMM's smooth model. Section 4 considers mean-preserving spreads in ambiguity perception. Result 1 shows that a mean-preserving spread in ambiguity perception can actually lead to a lower VSL of an ambiguity averse decision maker. Result 2 shows that a general mean-preserving spread in ambiguity perception always leads to a higher VSL if ambiguity prudence is not too high. It highlights the importance of ambiguity prudence in the evaluation of mortality risk benefits.

\footnotetext{
${ }^{1}$ Viscusi (2014) provides an overview of government practices and lists VSL amounts used in different regulatory analyses.

2 See e.g. Mankiw and Zeldes (1991) and Haliassos and Bertaut (1995).

${ }^{3}$ French and Poterba (1991).
} 
Section 5 extends the analysis to higher order changes in ambiguity. We define the concept of $n^{\text {th }}$ order increases in ambiguity and show that, under the smooth model, the effect of these increases on the VSL depends on higher order ambiguity preferences.

The remainder of the paper explores the robustness of our findings. In Section 6, we consider the case of ambiguity seeking. While most of the theoretical literature has focused on ambiguity aversion, Ellsberg (1961) already pointed out that people may sometimes be ambiguity seeking, particularly for unlikely events and when losses are involved (for an overview of empirical evidence supporting Ellsberg's conjecture see Trautmann and van de Kuilen 2016; Wakker 2010). As the risk of death is typically small, the case of ambiguity seeking cannot be ignored. Result 5 shows that the combination of ambiguity seeking and ambiguity prudence makes it impossible to sign the effect of changes in ambiguity perception on the VSL. This is noteworthy as most ambiguity models imply ambiguity prudence (Baillon 2017).

Most of our analysis uses KMM's smooth model. The smooth model incorporates ambiguity aversion by using a different utility function for uncertainty than for risk. A different strand of the ambiguity literature models ambiguity aversion through a difference in the weighting of events between risk and uncertainty. The main models in this class are Choquet expected utility (Schmeidler 1989) and the multiple priors models (Gilboa and Schmeidler 1989). Baillon and Bleichrodt (2015) found that Choquet expected utility better described ambiguity preferences than the smooth model. In Section 7, we extend our analysis to Chateauneuf et al.'s (2007) neoadditive preferences, which have intuitive interpretations in terms of Choquet expected utility and in terms of multiple priors. We show that our conclusions remain valid under neo-additive preferences: ambiguity aversion alone does not necessarily lead to an increase in the VSL when perceived ambiguity increases and ambiguity prudence plays a key role.

\section{Background}

The standard VSL model (Drèze 1962) assumes that a decision maker (DM) evaluates decisions involving a fatality risk by (state-dependent) expected utility:

$$
V_{0}=(1-p) U_{l}(w)+p U_{d}(w)
$$

In Eq. (1), $p$ is the probability that the DM dies during the current period, $U_{l}(w)$ is the DM's utility of wealth if he survives the period and $U_{d}(w)$ is his utility of wealth if he does not survive (i.e. his utility of a bequest). If the DM has no bequest motive then $U_{d}(w)$ is zero for all wealth levels.

It is common to assume that the DM prefers more wealth to less $\left(U_{l}^{\prime}(w)>0\right.$ and $U_{d}^{\prime}(w)>0$ for all $\left.w\right)$, that he is risk averse $\left(U_{l}^{\prime \prime}(w)<0\right.$ and $U_{d}^{\prime \prime}(w)<0$ for all $w$ ), and that both the utility of wealth and the marginal utility of wealth are always higher when alive than when dead $\left(U_{l}(w)>U_{d}(w), U_{l}^{\prime}(w)>U_{d}^{\prime}(w)\right.$ for all $\left.w\right)$. The VSL is the marginal rate of substitution between wealth and mortality risk. It is obtained by totally differentiating Eq. (1) with respect to $p$ and $w$ holding expected utility constant: 


$$
V S L_{r}=\frac{d w}{d p}=\frac{U_{l}(w)-U_{d}(w)}{(1-p) U_{l}^{\prime}(w)+p U_{d}^{\prime}(w)}
$$

The subscript $r$ serves as a reminder that Eq. (2) concerns the case of risk where the mortality risk is objectively known. Under the assumptions made, $V S L_{r}$ is strictly positive and increases with wealth and with the mortality risk $p$. The positive relation between the VSL and the mortality risk has been coined the "dead anyway" effect by Pratt and Zeckhauser (1996). It expresses that, abstracting from bequest considerations, a DM who faces a high probability of death will be inclined to spend as much as he can on mortality risk reduction as he is unlikely to survive anyway.

\section{The smooth ambiguity model}

We will now explore the impact of ambiguity. Suppose the baseline mortality risk $p$ is no longer objectively known, but is ambiguous. We express this by adding a random variable $\tilde{\varepsilon}$, which reflects the DM's perceived ambiguity, to $p$ so that the new mortality risk becomes: $\tilde{p}=p+\tilde{\varepsilon}$. To avoid negative probabilities or probabilities larger than 1 , the support of $\tilde{\varepsilon}$ is restricted to $[-p, 1-p]$. We assume that the DM evaluates the mortality risk according to the smooth model of KMM (2005):

$$
W_{\tilde{\varepsilon}}=\varphi^{-1}\left(E\left(\varphi\left((1-\tilde{p}) U_{l}(w)+\tilde{p} U_{d}(w)\right)\right)\right) .
$$

In the smooth model the DM's ambiguity perception is modeled by a second order distribution $\tilde{p}$, which reflects his beliefs about the mortality risk. The (increasing) function $\varphi$ reflects the DM's ambiguity attitudes. If $\varphi$ is everywhere concave $\left(\varphi^{\prime \prime}<0\right)$ then the DM is (uniformly) ambiguity averse. If $\varphi$ is everywhere convex $\left(\varphi^{\prime \prime}>0\right)$ then the DM is (uniformly) ambiguity seeking, If $\varphi$ is linear $\left(\varphi^{\prime \prime}=0\right)$, the DM is ambiguity neutral and the smooth model is equivalent to subjective expected utility. Then the DM behaves according to Eq. (2) with $p=E(\tilde{p})$. In Eq. (3) we use the subscript $\tilde{\varepsilon}$ to emphasize that utility depends on the DM's ambiguity perception.

We assume that $E[\tilde{\varepsilon}]=0$ and, thus, $E[\tilde{p}]=p$. In other words, ambiguity leads to an increase in the spread of the distribution of mortality risks that the DM perceives as possible, but it does not lead to a systematic bias in the perceived risks. We discuss the effects of a systematic bias in the perceived risks in the Electronic Supplementary Material.

We obtain the VSL under the smooth ambiguity model by totally differentiating Eq. (3) with respect to $p$ and $w$. This gives:

$$
V S L_{\tilde{\varepsilon}}=\frac{d w}{d p}=\frac{\left(U_{l}(w)-U_{d}(w)\right) E\left[\varphi^{\prime}\left((1-\tilde{p}) U_{l}(w)+\tilde{p} U_{d}(w)\right)\right]}{E\left[\left((1-\tilde{p}) U_{l}^{\prime}(w)+\tilde{p} U_{d}^{\prime}(w)\right) \varphi^{\prime}\left((1-\tilde{p}) U_{l}(w)+\tilde{p} U_{d}(w)\right)\right]}
$$


Treich (2010) showed that an ambiguity-averse DM will have a higher VSL under ambiguity than under risk: $V S L_{\tilde{\varepsilon}}>V S L_{r}$. The intuition is that an ambiguity averse DM concentrates on the higher mortality risks and we noticed before that higher mortality risks increase the VSL as a result of the dead anyway effect. This also implies that an increase in ambiguity aversion (reflected by a more concave $\varphi$ ) increases $V S L_{\tilde{\varepsilon}}$.

\section{Changes in perceived ambiguity}

We now turn to the comparison between two ambiguous situations. Consider two situations for which the DM's levels of perceived ambiguity are described by the random variables $\tilde{\varepsilon}_{1}$ and $\tilde{\varepsilon}_{2}$. If we write $\widetilde{p}_{i}=p+\tilde{\varepsilon}_{i}, i=1,2$, Eq. (4) becomes:

$$
V S L_{\tilde{\varepsilon}_{i}}=\frac{d w}{d p}=\frac{\left(U_{l}(w)-U_{d}(w)\right) E\left[\varphi^{\prime}\left(\left(1-\widetilde{p}_{i}\right) U_{l}(w)+\widetilde{p}_{i} U_{d}(w)\right)\right]}{E\left[\left(\left(1-\widetilde{p}_{i}\right) U_{l}^{\prime}(w)+\widetilde{p}_{i} U_{d}^{\prime}(w)\right) \varphi^{\prime}\left(\left(1-\widetilde{p}_{i}\right) U_{l}(w)+\widetilde{p}_{i} U_{d}(w)\right)\right]}
$$

In this section we consider the case where $\tilde{\varepsilon}_{2}$ is a mean-preserving spread of $\tilde{\varepsilon}_{1}$. Higher order changes in ambiguity are studied in the next section. Because $\tilde{\varepsilon}_{2}$ is a mean-preserving spread of $\tilde{\varepsilon}_{1}, E\left[\tilde{\varepsilon}_{1}\right]=E\left[\tilde{\varepsilon}_{2}\right]$, but $\operatorname{Var}\left[\tilde{\varepsilon}_{1}\right]<\operatorname{Var}\left[\tilde{\varepsilon}_{2}\right]$. Treich $(2010)$ showed that if $\tilde{\varepsilon}_{1}=0 V S L_{\tilde{\varepsilon}_{2}}$ will exceed $V S L_{\tilde{\varepsilon}_{1}}$. The next example shows that this is no longer true when $\tilde{\varepsilon}_{1}$ and $\tilde{\varepsilon}_{2}$ are both nondegenerate.

Let $\varphi(x)=-\frac{e^{-\alpha x}}{\alpha}, \alpha>0$. We can measure the intensity of the DM's ambiguity aversion as $-\frac{\varphi^{\prime \prime}}{\varphi^{\prime}}$, much like the well-known Arrow-Pratt index measures the DM's risk aversion. The function $\varphi(x)=-\frac{e^{-\alpha x}}{\alpha}$ has the attractive property that ambiguity aversion is constant and equal to $\alpha$. Normalize wealth to 1 and let $U_{l}=\left(1-e^{-.1 x}\right)$ and $U_{d}=0.5$ $*\left(1-e^{-.05 x}\right)$. Then $U_{l}(x)>U_{d}(x)$ and $U_{l}^{\prime}(x)>U_{d}^{\prime}(x)$ for all positive wealth levels $x$. We write $\left(p_{1}: x_{1}, \ldots, p_{n}: x_{n}\right)$ for the random variable that gives $x_{j}$ with probability $p_{j}, j=$ $1, \ldots, n$. Consider two random variables $\tilde{\varepsilon}_{1}=(0.5:-0.2,0.5: 0.2)$ and $\tilde{\varepsilon}_{2}=(0.125:-$ $0.5,0.375:-0.1,0.5: 0.2)$. Then $E\left[\tilde{\varepsilon}_{1}\right]=E\left[\tilde{\varepsilon}_{2}\right]=0$ and $\tilde{\varepsilon}_{2}$ is a mean-preserving spread of $\tilde{\varepsilon}_{1}$. Figure 1 displays the relation between $V S L_{\tilde{\varepsilon}_{1}}-V S L_{\tilde{\varepsilon}_{2}}$ and $\alpha$. The figure shows that initially $V S L_{\tilde{\varepsilon}_{2}}$ exceeds $V S L_{\tilde{\varepsilon}_{1}}$, but as $\alpha$ increases the difference becomes smaller and for $\alpha$ sufficiently large the relation is reversed and $V S L_{\tilde{\varepsilon}_{2}}$ is less than $V S L_{\tilde{\varepsilon}_{1}}$. Because the intensity of the DM's ambiguity aversion is equal to $\alpha$, the figure shows that more ambiguity aversion does not necessarily lead to a higher VSL when the DM's ambiguity perception increases.

In other words, our example shows the following:

RESULT 1: Let $\tilde{\varepsilon}_{1}$ and $\tilde{\varepsilon}_{2}$ be two nondegenerate random variables with $\tilde{\varepsilon}_{2}$ a meanpreserving spread of $\tilde{\varepsilon}_{1}$. Then the sign of $V S L_{\tilde{\varepsilon}_{2}}-V S L_{\tilde{\varepsilon}_{1}}$ is indeterminate.

Why doesn't Treich's result carry over to the more general case where both risks are ambiguous? For notational convenience, let $\widetilde{Z}_{l}=\left(1-\tilde{p}_{i}\right) U_{l}(w)+\widetilde{p}_{l} U_{d}(w)$ and $\widetilde{Z}_{l}^{\prime}=\left(1-\tilde{p}_{i}\right) U_{l}^{\prime}(w)+\widetilde{p}_{l} U_{d}^{\prime}(w), i=1,2$. In the special case where one situation is 


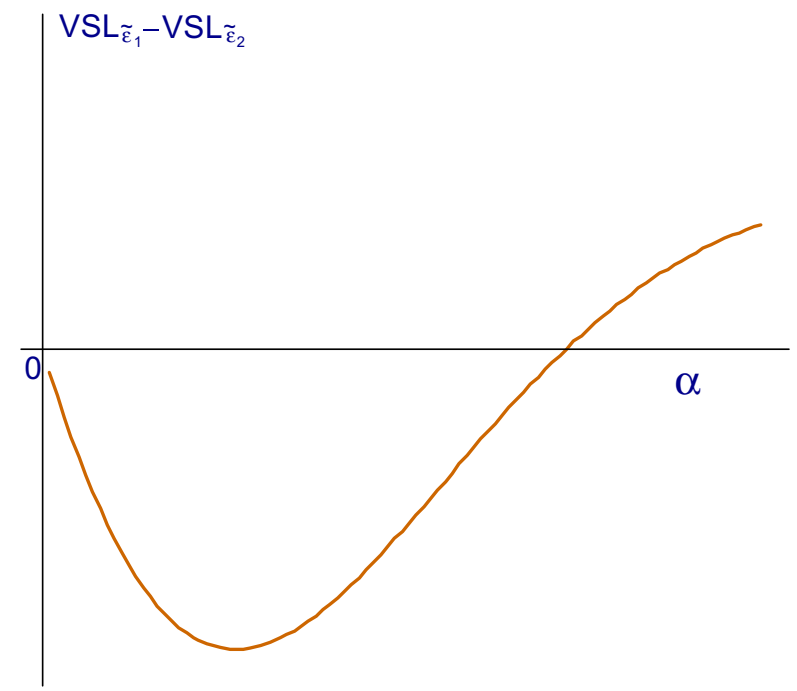

Fig. 1 The relationship between $V S L_{\tilde{\varepsilon}_{1}}-V S L_{\tilde{\varepsilon}_{2}}$ and ambiguity aversion (measured by the parameter $\alpha$ ) in the example

unambiguous $\left(\tilde{\varepsilon}_{1}=0\right), \tilde{Z}_{1}^{\prime}$ is constant. Then the denominator of Eq. (5) becomes $E\left[\tilde{Z}_{1}^{\prime}\right] E\left[\varphi^{\prime}\left(\tilde{Z}_{1}\right)\right]$. Because $\widetilde{\varepsilon_{2}}$ is a mean-preserving spread of $\tilde{\varepsilon}_{1}, E\left[\tilde{Z}_{1}^{\prime}\right]=E\left[\tilde{Z}_{2}^{\prime}\right]$. Substituting $E\left[\tilde{Z}_{1}^{\prime} \varphi^{\prime}\left(\tilde{Z}_{1}\right)\right]=E\left[\tilde{Z}_{1}^{\prime}\right] E\left[\varphi^{\prime}\left(\tilde{Z}_{1}\right)\right]$ and $E\left[\tilde{Z}_{1}^{\prime}\right]=E\left[\tilde{Z}_{2}^{\prime}\right]$ into Eq. (5) gives after some rearranging that $V S L_{\tilde{\varepsilon}_{2}}>V S L_{\tilde{\varepsilon}_{1}}$ iff $E\left[\tilde{Z}_{2}^{\prime}\right] E\left[\varphi^{\prime}\left(\tilde{Z}_{2}\right)\right]>E\left[\tilde{Z}_{2}^{\prime} \varphi^{\prime}\left(\tilde{Z}_{2}\right)\right]$. This last inequality holds if the covariance between $\tilde{Z}_{2}^{\prime}$ and $\varphi^{\prime}\left(\tilde{Z}_{2}\right)$ is negative, which is true for an ambiguity averse DM. However, if $\tilde{\varepsilon}_{1}$ is also ambiguous, $\tilde{Z}_{1}^{\prime}$ is no longer constant and the above argument can no longer be applied.

To ensure that a mean-preserving spread in ambiguity always leads to an increase in the VSL, we must impose additional conditions on the DM's ambiguity attitudes, in particular ambiguity prudence. Result 2 gives sufficient conditions. Baillon (2017) gives a model-free definition of ambiguity prudence. Ambiguity prudence is implied by most ambiguity models. It reflects the intuition that a decision maker prefers to spread harms across events rather than to concentrate them in one or a few events. In our decision context, it means that the DM prefers to have ambiguity in the mortality risk in states of the world in which the mortality risk is low rather than in states of the world in which the mortality risk is high. The importance of prudence in explaining economic behavior is widely-documented for decisions under risk where it corresponds to a preference for precautionary saving (Kimball 1990). For ambiguity, Guerdjikova and Sciubba (2015) show that ambiguity prudence plays a crucial role in the survival of ambiguity averse agents in the market. Baillon (2017) illustrates its importance for prevention behavior. Other recent illustrations of the role of ambiguity prudence are Berger $(2014,2016)$ on saving and prevention, Gierlinger and Gollier (2015) on the socially efficient discount rate, and Peter and Ying (forthcoming) on insurance.

Under the smooth model, ambiguity prudence is equivalent to $\varphi^{\prime \prime \prime}>0$. We can define an index of ambiguity-prudence as $-\frac{\varphi^{\prime \prime \prime}}{\varphi^{\prime \prime}}$ with higher values of the index 
corresponding to more ambiguity prudence. The index reflects the extent to which the DM cares about the skewness of his ambiguity perceptions. If $\varphi^{\prime \prime \prime}=0$, the DM does not care about skewness. If $\varphi^{\prime \prime \prime}<0$, the DM is ambiguity imprudent.

RESULT 2: For all $\tilde{\varepsilon}_{1}$ and $\tilde{\varepsilon}_{2}$ with $\tilde{\varepsilon}_{2}$ a mean-preserving spread of $\tilde{\varepsilon}_{1}$,

i. If the DM is ambiguity prudent and $-\frac{\varphi^{\prime \prime \prime}}{\varphi^{\prime \prime}}<2 S^{*}$ then $V S L_{\tilde{\varepsilon}_{2}}>V S L_{\tilde{\varepsilon}_{1}}$.

ii. If $\varphi^{\prime \prime \prime}=0$ then also $V S L_{\tilde{\varepsilon}_{2}}>V S L_{\tilde{\varepsilon}_{1}}$.

iii. If the DM is ambiguity imprudent then the sign of $V S L_{\tilde{\varepsilon}_{2}}-V S L_{\tilde{\varepsilon}_{1}}$ is indeterminate.

Proofs of all results presented throughout the paper are in the Electronic Supplementary Material. There we also explain why in case (iii) the sign of $V S L_{\tilde{\varepsilon}_{2}}-V S L_{\tilde{\varepsilon}_{1}}$ is indeterminate.

In Result 2 part (i), $S^{*}$ denotes the minimum value of $S=\frac{U_{l}^{\prime}-U_{d}^{\prime}}{\left(U_{l}-U_{d}\right)\left(Z_{\mathrm{i}}^{\prime}-\varepsilon_{i}\left(U_{l}^{\prime}-U_{d}^{\prime}\right)\right)}, i=1$, 2. It is obtained for the minimum value that the random variable $\tilde{\varepsilon}_{2}$ takes as we show in the Electronic Supplementary Material. Result 2 part (ii) says that a mean-preserving spread in the DM's perceived ambiguity always leads to an increase in the VSL if the DM does not care about the skewness of the distribution of his perceived mortality risks. This is the only case where we can straightforwardly extend Treich's result. If the DM is ambiguity prudent then a mean-preserving spread in ambiguity perception also leads to an increase in the VSL if his ambiguity prudence is not too extreme (it should not exceed $S^{*}$ ). The reason why ambiguity prudence cannot be too extreme is to exclude cases like the one in our example. For $\varphi(x)=-\frac{e^{-\alpha x}}{\alpha}$ the index of ambiguity prudence is equal to $\alpha$ and the example shows that if ambiguity prudence becomes too large then Result 2 part (i) no longer holds. Intuitively, $\tilde{\varepsilon}_{2}$ can be more negatively-skewed than $\tilde{\varepsilon}_{1}$. An ambiguity prudent DM likes this negative skewness. On the other hand, the DM is also ambiguity averse and dislikes the greater ambiguity involved in $\tilde{\varepsilon}_{2}$. If his ambiguity prudence is not too strong, the negative effect of ambiguity aversion will dominate and the increase in ambiguity leads to an increase in the VSL. However, if ambiguity prudence is strong then the positive effect of the negative skewness of $\tilde{\varepsilon}_{2}$ can prevail and the VSL will decrease.

\section{Generalization to higher order changes in ambiguity}

We will now extend our analysis to more general changes in ambiguity perception. Consider, as before, two random variables $\tilde{\varepsilon}_{1}$ and $\tilde{\varepsilon}_{2}$ with $\tilde{p}_{i}=p+\tilde{\varepsilon}_{i}, i=1,2$. Let $F_{i}$ be the cumulative probability distribution of $\tilde{p}_{i}$. $[c, d]$ denotes the support of $\tilde{p}_{1} \cup \tilde{p}_{2}$, i.e. the DM believes that mortality risks outside the interval $[c, d]$ are impossible and, thus, $F_{i}(c)=0$ and $F_{i}(d)=1, i=1,2$. Obviously, $[c, d]$ is a subset of [0,1], as probabilities cannot be negative or exceed 1 .

Rewrite $F_{i}^{0}=\tilde{p}_{i}, F_{i}^{1}=F_{i}, i=1,2$ and define repeated integrals $F_{i}^{k}$ for $k \geq 1$ by:

$$
F_{i}^{k}(p)=\int_{c}^{p} F_{i}^{k-1}(q) d q
$$

For decision under risk, Ekern (1980) gave a definition of more $n^{\text {th }}$ order risk aversion when risks about wealth are introduced. Caballé and Pomansky (1996) defined a DM 
as mixed risk averse if his von Neumann-Morgenstern utility function $u$ has positive odd and negative even derivatives: for all $k=1, \ldots, n,(-1)^{(k+1)} u^{(k)}>0$. Ekern's (1980) definition implies that a mixed risk averse DM will find any $n^{\text {th }}$ order increase in risk undesirable.

Under the smooth model, we define a DM as mixed ambiguity averse if his ambiguity function $\varphi$ has positive odd and negative even derivatives: for all $k=1, \ldots, n$, $(-1)^{(k+1)} \varphi^{(k)}>0$. Courbage and Rey (2016) defined a change in ambiguity $\tilde{\varepsilon}_{2}$ as more ambiguous than another change $\tilde{\varepsilon}_{1}$ if every mixed ambiguity averse DM prefers $\tilde{p}_{1}=p+\tilde{\varepsilon}_{1}$ to $\tilde{p}_{2}=p+\tilde{\varepsilon}_{2}$. Using this definition, we can now formally define what it means to have more $n^{\text {th }}$ order ambiguity in the context of our decision problem.

Definition $1 \tilde{\varepsilon}_{2}$ has more $n^{\text {th }}$ order ambiguity than $\tilde{\varepsilon}_{1}$, written $\tilde{\varepsilon}_{2} \succcurlyeq_{n} \tilde{\varepsilon}_{1}$ if

i. $\quad F_{2}^{k}(d)=F_{1}^{k}(d)$ for $k=1, \ldots, n$

ii. If $n$ is odd, $F_{2}^{n}(p) \leq F_{1}^{n}(p)$ for all $p \in[c, d]$ and there exists a $p \in[c, d]$ for which $F_{2}^{n}(p)<F_{1}^{n}(p)$. If $n$ is even, $F_{2}^{n}(p) \geq F_{1}^{n}(p)$ for all $p \in[c, d]$ and there exists a $p \in[c, d]$ for which $F_{2}^{n}(p)>F_{1}^{n}(p)$.

Part (i) of Definition 1 implies that the $(n-1)$ first moments of $F_{1}$ and $F_{2}$ are equal. Part (ii) implies that the $n^{\text {th }}$ moment of $F_{2}$ exceeds the $n^{\text {th }}$ moment of $F_{1} \cdot{ }^{4}$ So if $\tilde{\varepsilon}_{2}$ has more first order ambiguity than $\tilde{\varepsilon}_{1}$ then the mean of $\tilde{\varepsilon}_{2}$ exceeds the mean of $\tilde{\varepsilon}_{1}$. In other words, $\tilde{\varepsilon}_{2}$ has a higher expected mortality risk than $\tilde{\varepsilon}_{1}$. If $\tilde{\varepsilon}_{2}$ has more second order ambiguity than $\tilde{\varepsilon}_{1}$ then $\tilde{\varepsilon}_{2}$ is a mean-preserving spread of $\tilde{\varepsilon}_{1}$, the case we considered in Section 4. If $\tilde{\varepsilon}_{2}$ has more third order ambiguity than $\tilde{\varepsilon}_{1}$ then $\tilde{\varepsilon}_{2}$ can be obtained from $\tilde{\varepsilon}_{1}$ by a series of mean-variance-preserving-transformations (Menezes et al. 1980). These transformations do not affect the mean and the variance but they transfer ambiguity from lower to higher values of the mortality risk with the result that the distribution becomes more positively skewed.

Lemma A3 in the Electronic Supplementary Material shows that a mixed ambiguity averse DM will indeed always dislike increases in $n^{\text {th }}$ order ambiguity. The intuition is that a mixed ambiguity averse DM prefers to distribute harms across states of nature rather than to concentrate them in one state. In other words, he prefers to combine good with bad over combining good with good (and bad with bad). An example may clarify. Suppose a DM faces a certain increase $k$ in his perceived mortality risk and a zero mean random change in ambiguity perception $\tilde{\varepsilon}$. All ambiguity averse DMs will dislike these two changes and they are both perceived as harmful. A mixed ambiguity averse DM will then prefer a situation in which his ambiguity perception changes to $[0.5: k ; 0.5: \tilde{\varepsilon}]$ to the situation in which it changes to $[0.5: k+\tilde{\varepsilon} ; 0.5: 0]$. In the latter situation, the two harms are concentrated in one state, whereas in the former situation they are divided over the two states. The two changes have the same mean $(0.5 k)$ and the same variance $\left(0.5 k^{2}\right)$, but $[0.5: k+\tilde{\varepsilon} ; 0.5: 0]$ is more positively skewed. Definition 1 reflects that a mixed ambiguity averse DM dislikes such positive skewness.

\footnotetext{
${ }^{4}$ We introduce the distinction between $n$ odd and $n$ even to make sure that a decision maker who is mixed ambiguity averse always prefers $\tilde{\varepsilon}_{1}$ to $\tilde{\varepsilon}_{2}$. Our definition is slightly different from that of Ekern (1980) who considers random changes in wealth, with more wealth always desirable, while we consider random changes in the mortality risk, increases of which are undesirable.
} 
The assumption of mixed ambiguity aversion is plausible and common in the literature. Brockett and Golden (1987) have pointed out that all commonly used functions in economic theory with a positive first derivative and a negative second derivative have successive derivatives that change sign. Consequently, all functions $\varphi$ that reflect ambiguity aversion must be mixed ambiguity averse. Examples of functions that are mixed ambiguity averse are the constant ambiguity aversion function $\varphi(x)=-$ $e^{-\alpha x} / \alpha$, for $\alpha>0$, which we used in our example and which was also used by KMM (2005), and the functions used in Ju and Miao (2012) and in Gollier (2011).

We can now state the generalization of Result 2 to $n^{\text {th }}$ order changes in ambiguity.

Result 3. Let the DM be mixed ambiguity averse. For all $\tilde{\varepsilon}_{1}$ and $\tilde{\varepsilon}_{2}$ such that $\tilde{\varepsilon}_{2} \succcurlyeq_{n} \tilde{\varepsilon}_{1}$, if $-\frac{\varphi^{(n+1)}}{\varphi^{(n)}}<n S^{*}$ then $V S L_{\tilde{\varepsilon}_{2}}>V S L_{\tilde{\varepsilon}_{1}}$.

$S^{*}$ is defined as the minimum of $S=\frac{U_{l}^{\prime}-U_{d}^{\prime}}{\left(U_{l}-U_{d}\right)\left(Z_{i}^{\prime}-\varepsilon_{i}\left(U_{l}^{\prime}-U_{d}^{\prime}\right)\right)}$. In words, Result 3 says that if the DM's $n^{\text {th }}$ order ambiguity aversion is not too extreme, $n^{\text {th }}$ order increases in ambiguity will lead to an increase in his VSL.

\section{Ambiguity seeking}

Until now, we have only analyzed the preferences of an ambiguity averse DM. However, empirical evidence suggests that uniform ambiguity aversion is rare and that there are decision contexts in which ambiguity seeking prevails (Kocher et al. 2018; Trautmann and van de Kuilen 2016; Wakker 2010). This holds in particular for unlikely events and losses.

In the smooth model an ambiguity seeking DM is characterized by $\varphi^{\prime}>0$ and $\varphi^{\prime \prime}>0$. The introduction of ambiguity increases the utility of an ambiguity seeker and Result 4 shows that it lowers the VSL of an ambiguity seeking DM compared with the situation in which there is no ambiguity:

RESULT 4: Let the DM be ambiguity seeking. Let $\tilde{\varepsilon}_{1}$ and $\tilde{\varepsilon}_{2}$ be two random variables with $\tilde{\varepsilon}_{1}=0$ and $\tilde{\varepsilon}_{2}$ a mean-preserving spread of $\tilde{\varepsilon}_{1}$. Then $V S L_{\tilde{\varepsilon}_{2}}<V S L_{\tilde{\varepsilon}_{1}}$.

Result 5 summarizes what happens if $\tilde{\varepsilon}_{2}$ is a mean-preserving spread of $\tilde{\varepsilon}_{1}$ and both situations are ambiguous (i.e., both $\tilde{\varepsilon}_{1}$ and $\tilde{\varepsilon}_{2}$ are nondegenerate). It is the immediate counterpart of Result 2.

Result 5: Let the DM be ambiguity seeking. For all $\tilde{\varepsilon}_{1}$ and $\tilde{\varepsilon}_{2}$ with $\tilde{\varepsilon}_{2}$ a meanpreserving spread of $\tilde{\varepsilon}_{1}$,

i. If the DM is ambiguity imprudent and $-\frac{\varphi^{\prime \prime \prime}}{\varphi^{\prime \prime}}<2 S^{*}$ then $V S L_{\tilde{\varepsilon}_{2}}<V S L_{\tilde{\varepsilon}_{1}}$.

ii. If $\varphi^{\prime \prime \prime}=0$ then also $V S L_{\tilde{\varepsilon}_{2}}<V S L_{\tilde{\varepsilon}_{1}}$.

iii. If the DM is ambiguity prudent then the sign of $V S L_{\tilde{\varepsilon}_{2}}-V S L_{\tilde{\varepsilon}_{1}}$ is indeterminate.

In part (i), $S^{*}$ is defined as in Result 2. 
Finally, Result 6 summarizes what happens in the case of higher order increases in ambiguity. While Results 4 and 5 are rather straightforward counterparts of Results 1 and 2, Result 6 is a bit different. The intuition underlying this difference is that, unlike for an ambiguity averse DM, for an ambiguity seeking DM the first and second derivatives of $\varphi$ do not change sign.

Result 6. Let the DM be ambiguity seeking. For all $\tilde{\varepsilon}_{1}$ and $\tilde{\varepsilon}_{2}$ such that $\tilde{\varepsilon}_{2} \succcurlyeq_{n} \tilde{\varepsilon}_{1}$ with $n \geq 3$ :

i. If $\varphi^{(n)}>0$ and $\varphi^{(n+1)}<0$ for $n$ odd and if $-\frac{\varphi^{(n+1)}}{\varphi^{(n)}}<n S^{*}$ then $V S L_{\tilde{\varepsilon}_{2}}>V S L_{\tilde{\varepsilon}_{1}}$. ii. If $\varphi^{(n)}>0$ and $\varphi^{(n+1)}<0$ for $n$ even and if $-\frac{\varphi^{(n+1)}}{\varphi^{(n)}}<n S^{*}$ then $V S L_{\tilde{\varepsilon}_{2}}<V S L_{\tilde{\varepsilon}_{1}}$.

with $S^{*}$ as in Result 3.

In words, we get conclusive results for an ambiguity seeker if the higher order derivatives change sign and if the ratio of the derivatives is not too extreme. In all other cases no conclusive results can be derived. Analogously to the case of mixed risk seeking (Crainich et al. 2013), we say that a DM is mixed ambiguity seeking if the derivatives of his ambiguity function $\varphi$ are always positive: $\varphi^{(n)}>0$ for all $n$. Mixed ambiguity seeking can be explained by a preference for combining good with good. Result 6 shows that we can make no clear predictions about the effect of $n^{\text {th }}$ order increases in ambiguity for mixed ambiguity seekers.

\section{Neo-additive preferences}

The empirical literature gives no clear answer which ambiguity model best describes people's preferences. While the results in Cubitt et al. (forthcoming) are consistent with the smooth model, Baillon and Bleichrodt (2015) and Chew et al. (2017) observed that models like Choquet expected utility (Schmeidler 1989) and $\alpha$-maxmin (Ghirardato et al. 2004) could better explain their data. In this section we explore the robustness of our results under the neo-additive model of Chateauneuf et al. (2007). Neo-additive preferences are a special case of Choquet expected utility and they also have an interpretation in terms of $\alpha$-maxmin (see for instance Baillon et al. 2017). Under neo-additive preferences, the DM's evaluation of the mortality risk is equal to: ${ }^{5}$

$$
\begin{aligned}
W_{\tilde{\varepsilon}}= & (1-a) E\left((1-\tilde{p}) U_{l}(w)+\tilde{p} U_{d}(w)\right)+\frac{a-b}{2} \max \left[(1-\tilde{p}) U_{l}(w)+\tilde{p} U_{d}(w)\right] \\
& +\frac{a+b}{2} \min \left[(1-\tilde{p}) U_{l}(w)+\tilde{p} U_{d}(w)\right] .
\end{aligned}
$$

In Eq. (7) $a \in[0,1]$ and $b \in[-a, a]$. A neo-additive DM gives weight $(1-a)$ to the expected utility of a random variable $\tilde{p}$, weight $\frac{a-b}{2}$ to the maximum (expected) utility that he can obtain and weight $\frac{a+b}{2}$ to the minimum (expected) utility that he can obtain. Expected utility is the special case of Eq. (7) with $a=b=0$. Baillon (2017) shows that

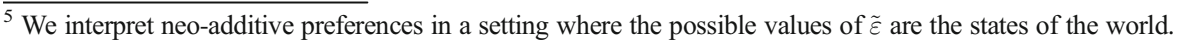


under neo-additive preferences his model-free definition of ambiguity aversion is equivalent to $b>0$ and his definition of ambiguity prudence to $a>0$. Hence, neoadditive preferences provide an easy way to capture ambiguity aversion and ambiguity prudence.

For a random variable $\tilde{\varepsilon}$ the maximum expected utility is obtained for the lowest mortality risk, i.e. for the lowest value of $\tilde{\varepsilon}$. Denote the absolute value of this by $\varepsilon_{\text {min }}$. Similarly, the minimum expected utility is obtained for the highest mortality risk, i.e. for the highest value of $\tilde{\varepsilon}$. Denote the absolute value of this by $\varepsilon_{\max }$. As we consider changes in ambiguity perception with mean zero, Eq. (7) can then be written as:

$$
\begin{aligned}
& (1-a)\left((1-p) U_{l}(w)+p U_{d}(w)\right)+\frac{a-b}{2}\left[\left(1-\left(p-\varepsilon_{\min }\right)\right) U_{l}(w)+\left(p-\varepsilon_{\min }\right) U_{d}(w)\right] \\
& \quad+\frac{a+b}{2}\left[\left(1-\left(p+\varepsilon_{\max }\right)\right) U_{l}(w)+\left(p+\varepsilon_{\max }\right) U_{d}(w)\right]
\end{aligned}
$$

Totally differentiating Eq. (8) with respect to $p$ and $w$ gives the VSL under neo-additive preferences:

$$
V S L_{\tilde{\varepsilon}_{i}}=\frac{d w}{d p}=\frac{U_{l}(w)-U_{d}(w)}{(1-p) U_{l}^{\prime}(w)+p U_{d}^{\prime}(w)+\left(U_{l}^{\prime}(w)+U_{d}^{\prime}(w)\right)\left[\frac{a-b}{2} \varepsilon_{\min }-\frac{a+b}{2} \varepsilon_{\max }\right]}
$$

As $a \in[0,1]$ and $b \in[-a, a]$, it follows that if the DM is ambiguity averse $(b>0)$ the sign of the term $\left[\frac{a-b}{2} \varepsilon_{\min }-\frac{a+b}{2} \varepsilon_{\max }\right]$ is indeterminate and depends on the relative magnitudes of $\varepsilon_{\min }$ and $\varepsilon_{\max }$. Hence, under neo-additive preferences we cannot replicate Treich's result that an ambiguity averse DM will always have a higher VSL under ambiguity than under no ambiguity. Two points are worth making. First, if $\varepsilon_{\min }=\varepsilon_{\max }$ then ambiguity aversion implies that $\left[\frac{a-b}{2} \varepsilon_{\min }-\frac{a+b}{2} \varepsilon_{\max }\right]$ is negative and that ambiguity leads to an increase in the VSL. Second, under Schmeidler's (1989) definition of ambiguity aversion, ${ }^{6} a=b$ and an ambiguity averse DM will always have a higher VSL when his perception of ambiguity increases.

Let us now consider what happens if the DM's ambiguity perception increases. Consider two random variables $\tilde{\varepsilon}_{1}$ and $\tilde{\varepsilon}_{2}$ with $\tilde{\varepsilon}_{2}$ a mean-preserving spread of $\tilde{\varepsilon}_{1}$. We denote the absolute values of the minima of $\tilde{\varepsilon}_{1}$ and $\tilde{\varepsilon}_{2}$ by $\varepsilon_{1, \min }$ and $\varepsilon_{2}$, min and their maximum values by $\varepsilon_{1, \max }$ and $\varepsilon_{2, \max }$.

RESUlT 7: Suppose that the DM has neo-additive preferences and is ambiguity averse $(b>0)$. Let $\tilde{\varepsilon}_{1}$ and $\tilde{\varepsilon}_{2}$ be two random variables with $\tilde{\varepsilon}_{2}$ a mean-preserving spread of $\tilde{\varepsilon}_{1}$.

i. if $\varepsilon_{2, \min }-\varepsilon_{1, \min } \leq \varepsilon_{2, \max }-\varepsilon_{1, \max }$ then $V S L_{\tilde{\varepsilon}_{2}}>V S L_{\tilde{\varepsilon}_{1}}$.

ii. if $\varepsilon_{2, \min }-\varepsilon_{1, \min }=e+k>\varepsilon_{2, \max }-\varepsilon_{1, \max }=e$ then $V S L_{\tilde{\varepsilon}_{2}}>V S L_{\tilde{\varepsilon}_{1}}$ if $a<\frac{2 e+k}{k} b$.

\footnotetext{
${ }^{6}$ Schmeidler (1989) defines ambiguity aversion as a preference for hedging: if the DM is indifferent between two random variables $\tilde{p}$ and $\tilde{q}$ then he prefers their mixture $\lambda \tilde{p}+(1-\lambda \tilde{q}), 0<\lambda<1$, to each of these variables.
} 
Result 7 shows that the effect of an increase in ambiguity perception depends on the skewness of the change in ambiguity perception and on the DM's ambiguity prudence. If $\varepsilon_{2, \min }-\varepsilon_{1, \min } \leq \varepsilon_{2, \max }-\varepsilon_{1, \max }$ then VSL will always increase after an increase in ambiguity perception. If $\varepsilon_{2, \min }-\varepsilon_{1, \min }$ exceeds $\varepsilon_{2, \max }-\varepsilon_{1, \max }$, but the DM is not too ambiguity prudent ( $a$ is not too high), then the VSL will increase after an increase in ambiguity perception. If ambiguity prudence is strong compared to ambiguity aversion and $\varepsilon_{2, \min }-\varepsilon_{1, \min }$ exceeds $\varepsilon_{2, \max }-\varepsilon_{1, \max }$ then it is possible that an increase in ambiguity perception actually leads to a decrease in the VSL. Result 7 also shows that the probability that $V S L_{\tilde{\varepsilon}_{2}}>V S L_{\tilde{\varepsilon}_{1}}$ increases if the DM becomes more ambiguity averse ( $b$ increases ceteris paribus).

Treich (2010) studied the special case of Result 7 where $\tilde{\varepsilon}_{1}=0$. By setting $\varepsilon_{2, \max }=\varepsilon_{\text {max }}, \varepsilon_{2, \min }=\varepsilon_{\text {min }}, \varepsilon_{1, \max }=\varepsilon_{1, \min }=0$, it follows that an ambiguity averse DM will always have a higher VSL under ambiguity than under no ambiguity if $a<\frac{\varepsilon_{\max }+\varepsilon_{\min }}{\varepsilon_{\min }-\varepsilon_{\max }} b .^{7}$

Result 8 states the effects of changes in ambiguity perception for an ambiguity seeking DM.

Result 8: Suppose that the DM has neo-additive preferences and is ambiguity seeking $(b<0)$. Let $\tilde{\varepsilon}_{1}$ and $\tilde{\varepsilon}_{2}$ be two random variables with $\tilde{\varepsilon}_{2}$ a mean-preserving spread of $\tilde{\varepsilon}_{1}$.

i. if $\varepsilon_{2, \min }-\varepsilon_{1, \min } \geq \varepsilon_{2, \max }-\varepsilon_{1, \max }$ then $V S L_{\tilde{\varepsilon}_{2}}>V S L_{\tilde{\varepsilon}_{1}}$.

ii. if $\varepsilon_{2, \min }-\varepsilon_{1, \min }=e<\varepsilon_{2, \max }-\varepsilon_{1, \max }=e+k$ then $V S L_{\tilde{\varepsilon}_{2}}<V S L_{\tilde{\varepsilon}_{1}}$ if $a<-\frac{2 e+k}{k} b$.

Hence, we observe, as in Result 5, that an ambiguity seeking DM's VSL will decrease when his ambiguity perception increases if he is not too ambiguity prudent.

\section{Conclusion}

The VSL is a key parameter in the evaluation of government regulation. The properties of the VSL have mainly been studied under risk where probabilities are objectively known. However, in most real-world decisions probabilities are at best vaguely known. The empirical literature shows that people are not neutral towards such ambiguity. Treich (2010) derived that an ambiguity averse DM who behaves according to the smooth ambiguity model of Klibanoff et al. (2005) will have a higher VSL under ambiguity than under no ambiguity. We have shown that this result cannot be extended to general increases in ambiguity. An ambiguity averse DM's VSL may actually be lower in more ambiguous decision situations. To sign the effect of increases in ambiguity, information on higher order ambiguity attitudes is required. We have particularly highlighted the important role of ambiguity prudence. Our results confirm Baillon's (2017) conclusion that ambiguity prudence plays a key role in explaining economic behavior.

\footnotetext{
$\overline{{ }^{7} \text { If } \tilde{\varepsilon} \text { is symmetric }\left(\varepsilon_{\min }=\varepsilon_{\max }\right)}$ or $\varepsilon_{\min }<\varepsilon_{\max }$ then it is of course always true that $V S L_{\tilde{\varepsilon}}>V S L_{r}$.
} 
The implications of our results for cost-benefit analysis depend on whether or not deviations from ambiguity neutrality are considered normative. Cost-benefit analysis is a prescriptive exercise, but the VSL is estimated by eliciting people's preferences for mortality risk reductions, which is a descriptive task. If ambiguity aversion is viewed as irrational then our results indicate what the bias in the estimated VSL will be. If an increase in perceived ambiguity is positively skewed, i.e., people perceive more situations with a high risk to human life, then they will react too strongly to the ambiguity leading to estimates of the VSL that are too high. An example is the approval of new pharmaceuticals (Viscusi and Zeckhauser 2015). If the distribution is negatively skewed, i.e., people perceive more situations in which the risk to human life is lower, and they are not too ambiguity prudent, their estimated VSL will be too low. On the other hand, if ambiguity aversion is considered rational (see for example Gilboa and Marinacci 2013) our results guide policy as to how the VSL used in policy evaluations should be adjusted to changes in ambiguity.

Empirical research on ambiguity prudence is still thin on the ground. Baillon et al. (2018) tested ambiguity prudence and found support for it. However, they only obtained qualitative support for ambiguity prudence and did not quantify its intensity. Our results highlight that such quantification is required to understand the effects of (increases in) ambiguity on the VSL. Ebert and Wiesen (2014) showed how the intensity of risk prudence can be measured. Extending their research to ambiguity is a worthwhile topic for future research.

Acknowledgements We are grateful to Kip Viscusi, Aurélien Baillon, and an anonymous reviewer for their comments on earlier versions of this paper. We thank Christina Stoddard for her careful proofreading. This research has benefited from the financial support of IDEXLYON from Université de Lyon (project INDEPTH) within the Programme Investissements d'Avenir (ANR-16-IDEX-0005).

Open Access This article is distributed under the terms of the Creative Commons Attribution 4.0 International License (http://creativecommons.org/licenses/by/4.0/), which permits unrestricted use, distribution, and reproduction in any medium, provided you give appropriate credit to the original author(s) and the source, provide a link to the Creative Commons license, and indicate if changes were made.

\section{References}

Andersson, H., \& Lindberg, G. (2009). Benevolence and the value of road safety. Accident Analysis and Prevention, 41(2), 286-293.

Baillon, A. (2017). Prudence with respect to ambiguity. Economic Journal, 127(604), 1731-1755.

Baillon, A., \& Bleichrodt, H. (2015). Testing ambiguity models through the measurement of probabilities for gains and losses. American Economic Journal: Micro, 7(2), 77-100.

Baillon, A., Bleichrodt, H., Keskin, U., l'Haridon, O., \& Li, C. (2017). Learning under ambiguity: An experiment using initial public offerings on a stock market. Management Science, 64(5), 2181-2198.

Baillon, A., Schlesinger, H., \& van de Kuilen, G. (2018). Measuring higher order ambiguity preferences. Experimental Economics, 21(2), 233-256.

Berger, L. (2014). Precautionary saving and the notion of ambiguity prudence. Economics Letters, 123(2), $248-251$.

Berger, L. (2016). The impact of ambiguity and prudence on prevention decisions. Theory and Decision, 80(3), 389-409.

Brockett, P., \& Golden, L. (1987). A class of utility functions containing all the common utility functions. Managing Science, 33, 955-964.

Caballé, J., \& Pomansky, A. (1996). Mixed risk aversion. Journal of Economic Theory, 71(2), 485-513. 
Chateauneuf, A., Eichberger, J., \& Grant, S. (2007). Choice under uncertainty with the best and worst in mind: Neo-additive capacities. Journal of Economic Theory, 137(1), 538-567.

Chew, S. H., Miao, B., \& Zhong, S. (2017). Partial ambiguity. Econometrica, 85(4), 1239-1260.

Courbage, C., \& Rey, B. (2016). On ambiguity apportionment. Journal of Economics, 118(3), 265-275.

Crainich, D., Eeckhoudt, L., \& Trannoy, A. (2013). Even (mixed) risk lovers are prudent. American Economic Review, 103(4), 1529-1535.

Cubitt, R., van de Kuilen, G., and S. Mukerji (forthcoming). Discriminating between Models of Ambiguity Attitude: a Qualitative Test, Journal of the European Economic Association.

Drèze, J. (1962). L'utilité sociale d'une vie humaine. Revue Française de Recherche Opérationnelle, 23, 93-118.

Ebert, S., \& Wiesen, D. (2014). Joint measurement of risk aversion, prudence, and temperance. Journal of Risk and Uncertainty, 48(3), 231-252.

Eeckhoudt, L., \& Hammitt, J. K. (2001). Background risks and the value of a statistical life. Journal of Risk and Uncertainty, 23(3), 261-279.

Ekern, S. (1980). Increasing nth degree risk. Economics Letters, 6(4), 329-333.

Ellsberg, D. (1961). Risk, ambiguity, and the Savage axioms. Quaterly Journal of Economics, 75(4), 643-669.

French, K. R., \& Poterba, J. M. (1991). Investor diversification and international equity markets. American Economic Review, 81(2), 222-226.

Ghirardato, P., Maccheroni, F., \& Marinacci, M. (2004). Differentiating ambiguity and ambiguity attitude. Journal of Economic Theory, 118(2), 133-173.

Gierlinger J., \& Gollier, C. (2015). Saving for an ambiguous future. Working Paper, UAB-Barcelona GSE \& MOV.

Gilboa, I., \& Schmeidler, D. (1989). Maxmin expected utility with a non-unique prior. Journal of Mathematical Economics, 18, 141-153.

Gilboa, I., \& Marinacci, M. (2016). Ambiguity and the Bayesian paradigm. In Readings in formal epistemology (pp. 385-439). Springer, Cham.

Gollier, C. (2011). Does ambiguity aversion reinforce risk aversion? Applications to portfolio choices and asset pricing. Review of Economic Studies, 78(4), 1329-1344.

Guerdjikova, A., \& Sciubba, E. (2015). Survival with ambiguity. Journal of Economic Theory, 155(1), 50-94.

Haliassos, M., \& Bertaut, C. C. (1995). Why do so few hold stocks? The Economic Journal, 105(432), 1110-1129.

Hammitt, J. K. (2000). Valuing mortality risk: Theory and practice. Environmental Science and Technology, 34(8), 1396-1400.

Hammitt, J. K. (2002). QALYs versus WTP. Risk Analysis, 22(5), 985-1001.

Hoy, M., Peter, R., \& Richter, A. (2014). Take-up for genetic tests and ambiguity. Journal of Risk and Uncertainty, 38(2), 111-133.

Ju, N., \& Miao, J. (2012). Ambiguity, learning and asset returns. Econometrica, 80(2), 559-591.

Kimball, M. S. (1990). Precautionary saving in the small and in the large. Econometrica, 58(1), 53-73.

Klibanoff, P., Marinacci, M., \& Mukerji, S. (2005). A smooth model of decision making under ambiguity. Econometrica, 73(6), 1849-1892.

Kocher, M. G., Lahno, A. M., \& Trautmann, S. T. (2018). Ambiguity aversion is not universal. European Economic Review, 101(1), 268-283.

Machina, M. J., \& Siniscalchi, M. (2014). Ambiguity and ambiguity aversion. In M. J. Machina \& W. K. Viscusi (Eds.), Handbook of the economics of risk and uncertainty (Vol. 1, pp. 729-807). Amsterdam: Elsevier.

Mankiw, N. G., \& Zeldes, S. P. (1991). The consumption of stockholders and nonstockholders. Journal of Financial Economics, 29(1), 97-112.

Menezes, C., Geiss, C., \& Tressler, J. (1980). Increasing downside risk. American Economic Review, 70(5), 921932.

Peter, R., \& Ying, J. (forthcoming). Do you Trust your Insurer? Ambiguity about Contract Nonperformance and Optimal Insurance Demand. Journal of Economic Behavior and Organization.

Pratt, J. W., \& Zeckhauser, R. J. (1996). Willingness to pay and the distribution of risk and wealth. Journal of Political Economy, 104(4), 747-763.

Ryan, M. J. (2009). Generalizations of SEU: A geometric tour of some non-standard models. Oxford Economic Papers, 61(2), 327-354.

Schmeidler, D. (1989). Subjective probability and expected utility without additivity. Econometrica, 57(3), 571-587.

Trautmann, S. T., \& van de Kuilen, G. (2016). Ambiguity attitudes. In G. Keren \& G. Wu (Eds.), Blackwell handbook of judgment and decision making (pp. 89-116). Chichester: Wiley Blackwell.

Treich, N. (2010). The value of a statistical life under ambiguity aversion. Journal of Environmental Economics and Management, 59(1), 15-26.

Viscusi, W. K. (2014). The value of individual and societal risks to life and health. In M. J. Machina \& W. K. Viscusi (Eds.), Handbook of the economics of risk and uncertainty (pp. 385-452). Amsterdam: Elsevier. 
Viscusi, W. K. (2018). Pricing lives: Guideposts for a safer society. Princeton University Press.

Viscusi, W. K., \& Aldy, J. E. (2003). The value of a statistical life: A critical review of market estimates throughout the world. Journal of Risk and Uncertainty, 27(1), 5-76.

Viscusi, W. K., \& Zeckhauser, R. J. (2015). Regulating ambiguous risks: The less than rational regulation of pharmaceuticals. The Journal of Legal Studies, 44(S2), S387-S422.

Wakker, P. (2010). Prospect theory for risk and ambiguity. Cambridge, UK: Cambridge University Press.

Publisher's note Springer Nature remains neutral with regard to jurisdictional claims in published maps and institutional affiliations. 